\title{
Ultrasonic hydrophone based on short in-fiber Bragg gratings
}

\author{
N. E. Fisher, D. J. Webb, C. N. Pannell, D. A. Jackson, L. R. Gavrilov, J. W. Hand, \\ L. Zhang, and I. Bennion
}

\begin{abstract}
We investigate the feasibility of using in-fiber Bragg gratings for measuring acoustic fields in the megahertz range. We found that the acoustic coupling from the ultrasonic field to the grating leads to the formation of standing waves in the fiber. Because of these standing waves, the system response is complex and, as we show, the grating does not act as an effective probe. However, significant improvement in its performance can be gained by use of short gratings coupled with an appropriate desensitization of the fiber. A noise-limited pressure resolution of $\approx 4.5 \times 10^{-3} \mathrm{~atm} / \sqrt{ } \mathrm{Hz}$ was found. (c) 1998 Optical Society of America
\end{abstract}

OCIS codes: $170.0170,170.7170$.

\section{Introduction}

There is a need for an assessment of the safety of ultrasound in the megahertz range for medical applications, ${ }^{1-3}$ owing to the trend toward increasing output powers from diagnostic ultrasound equipment $^{3}$ and the widening use of high-intensity ultrasonic fields in a range of other medical applications including ultrasound surgery, hyperthermia, and lithotripsy. ${ }^{4-7}$ Often the assessment of such fields is based on theoretical models of some complexity because the acoustic fields present in the body may arise from nonideal sources, and their propagation through heterogeneous tissues in the body is likely to be influenced by many factors. Hence a direct determination of them in vivo is of importance.

Conventional detection is most commonly achieved with piezoelectric devices. These include transducers made from (1) piezoceramics such as lead zirconate titanate (PZT) and (2) piezoelectric polymers such as polyvinylide difluoride. Both offer high sen-

N. E. Fisher, D. J. Webb (D. J. Webb@ukc.ac.uk), C. N. Pannell, and D. A. Jackson are with the Applied Optics Group, University of Kent at Canterbury, Canterbury, Kent CT2 7NR, UK. L. R. Gavrilov and J. W. Hand are with the Radiological Sciences Unit, Hammersmith Hospital, Du Cane Road, London W12 OHS, UK. L. Zhang and I. Bennion are with the Department of Electronic Engineering, Photonics Research Group, Aston University, Birmingham B4 7ET, UK.

Received 1 June 1998; revised manuscript received 21 September 1998 .

0003-6935/98/348120-09\$15.00/0

(C) 1998 Optical Society of America sitivities with, in the latter case, an improved acoustic impedance match to water. However, common to transducers fabricated from both types of material are a susceptibility to electromagnetic interference and signal distortion, and a reduced sensitivity caused by the electrical loading effects of the transducer leads. For overcoming these electrical problems optical fiber arrangements can be utilized, and several such schemes based on interferometric and polarimetric techniques have been reported. ${ }^{8-15}$ In addition, the fact that the diameter of a fiber is small means that these optical systems are (in principle) ideally suited for minimally invasive procedures.

Here we demonstrate that in-fiber Bragg gratings (IFBG's) ${ }^{16}$ also can be used to detect high-frequency ultrasonic fields. These devices offer distinct advantages such as ease of multiplexing, simultaneous measurement of temperature and strain, ${ }^{17}$ and potentially low cost. In our experiments, however, we found data that indicate that the acoustic coupling from the ultrasonic field to the grating-fiber can lead to the formation of standing waves in the fiber. Because of these standing waves, the system response is complex and the grating does not act as an effective ultrasonic probe. However, as we show, significant improvement in the grating's performance can be gained by the use of short gratings (less than half the acoustic wavelength in fused quartz) coupled with an appropriate desensitization of the fiber.

\section{Experiment}

The arrangement used to interrogate the grating is shown in Fig. 1. This utilized a ramped lithium nio- 


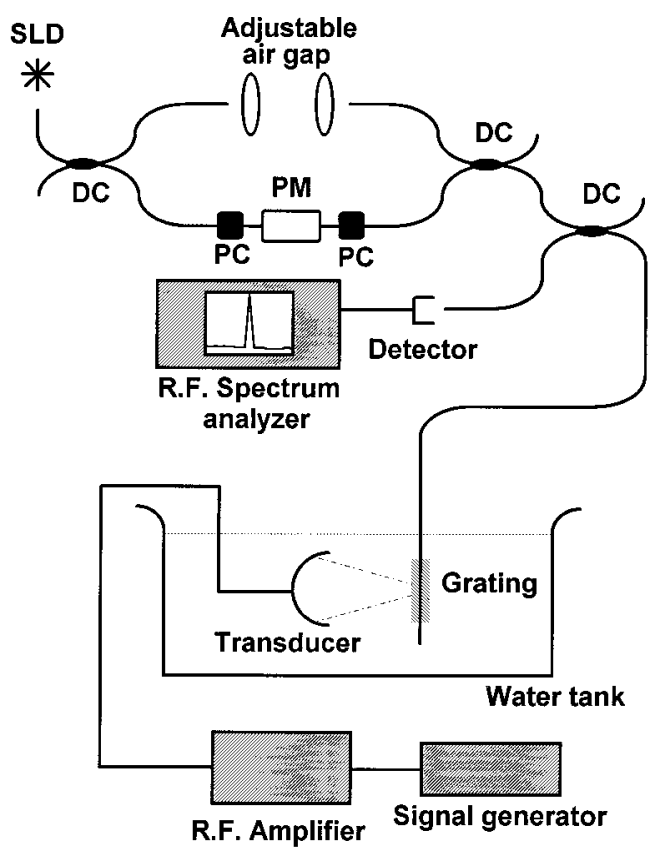

Fig. 1. Experimental arrangement: PM, phase modulator; PC, polarization controller; DC, directional coupler.

bate phase modulator (accurately set to produce a $2 \pi$ peak-to-peak phase excursion) to frequency shift the light in one arm of an unbalanced Mach-Zehnder interferometer (MZI) and thus allowed the use of heterodyne signal processing. ${ }^{18,19}$ Light from a pigtailed superluminescent diode (Superlum, Moscow), giving an output power of $1 \mathrm{~mW}$ centered at $824 \mathrm{~nm}$ with a bandwidth of $\approx 42 \mathrm{~nm}$, was launched into the unbalanced MZI; hence a channeled spectrum was created at the interferometer's outputs that was incident on the grating. Incorporated in one arm of the MZI was the phase modulator. The other arm contained a variable air gap that allowed the optical path difference (OPD) between the two arms to be adjusted. Provided that the OPD between the MZI's arms is longer than the source coherence length and shorter than the effective coherence length of the backreflected light from the grating, interference signals are observed at the detector, which can be expressed as

$$
I\left(\lambda_{B}\right)=A\left\{1+V \cos \left[\omega^{\prime} t+\Phi+\delta \Phi \sin \omega t+\phi(t)\right]\right\},
$$

where $\lambda_{B}$ is the wavelength of the reflected light from the modulated grating, $\omega^{\prime}$ is the angular frequency of the ramp modulation, $\omega$ is the angular frequency of the ultrasound incident on the grating, $A$ is proportional to the grating reflectivity, $V$ is the visibility of the signals, $\Phi=2 \pi \mathrm{OPD} / \lambda_{B}$, and $\phi(t)$ is a random phase-drift term. A strain-induced change in $\lambda_{B}$ from the grating, $\left(\delta \lambda_{B}\right)$, induces a change in phase shift in Eq. (1), given by

$$
\delta \Phi \sin \omega t=\left(2 \pi \mathrm{OPD} / \lambda_{B}{ }^{2}\right)\left(\delta \lambda_{B}\right) \sin \omega t .
$$

Hence from Eq. (1) strain-induced changes in $\lambda_{B}$ induce a corresponding phase modulation of the electrical carrier produced by the phase modulator,

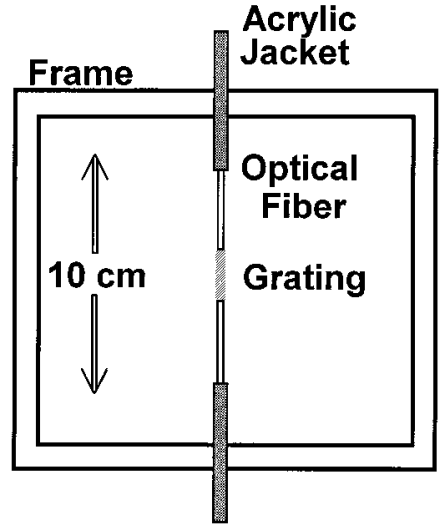

Fig. 2. Arrangement for the IFBG in a water tank.

which we measured by determining the amplitudes of the upper and lower sideband frequency components observed on a radio frequency spectrum analyzer.

The sensitivity of the system is determined by two competing effects. From Eq. (2) an increase in OPD results in a proportional increase in the amplitude of the phase modulation. On the other hand, increasing the OPD beyond the coherence length of the light reflected by the grating results in a reduction in the visibility and hence the height of the carrier and sidebands. In practice, we optimized the sensitivity by adjusting the OPD to obtain the maximum sideband amplitude.

The phase modulator was ramped, and hence generated a carrier signal, at $10 \mathrm{MHz}$. The grating had a nominal Bragg wavelength of $820 \mathrm{~nm}$, a bandwidth of $0.2 \mathrm{~nm}$, a reflectivity of $80 \%$, and a length of $5 \mathrm{~mm}$. A focused ultrasound piezoelectric transducer in the form of a section of a spherical shell was used as the source of ultrasound with a well-studied and predictable acoustic field distribution. ${ }^{4,5,20}$ Its parameters were as follows: resonant frequency, $1.91 \mathrm{MHz}$ (wavelength, $0.08 \mathrm{~cm}$ in water); piezoceramic material, PZT-4; diameter of piezoceramic plate, $6 \mathrm{~cm}$; and radius of curvature, $7 \mathrm{~cm}$.

The transducer was driven (in water) in continuous mode through a rf amplifier (Electronic Navigation Industries Model ENI 240L) and generated a maximum acoustic pressure of $\approx 2 \mathrm{~atm}$ (measured with a polyvinylide difluoride membrane hydrophone calibrated by the National Physical Laboratory, Teddington, Middlesex, UK) in a focal spot of 0.114-cm radius. This pressure corresponds to an acoustic intensity of $\approx 1.3 \mathrm{Wcm}^{-2}$. Finally, the grating-fiber was attached to a frame, as shown in Fig. 2, and positioned normally to the acoustic propagation direction.

\section{Results and Discussion}

\section{A. 5-mm Grating}

\section{Preliminary Results}

Consider first the spectrum analyzer trace of Fig. 3, which we obtained by modulating the grating at approximately $700 \mathrm{~Hz}$ using sound waves in air. Note 


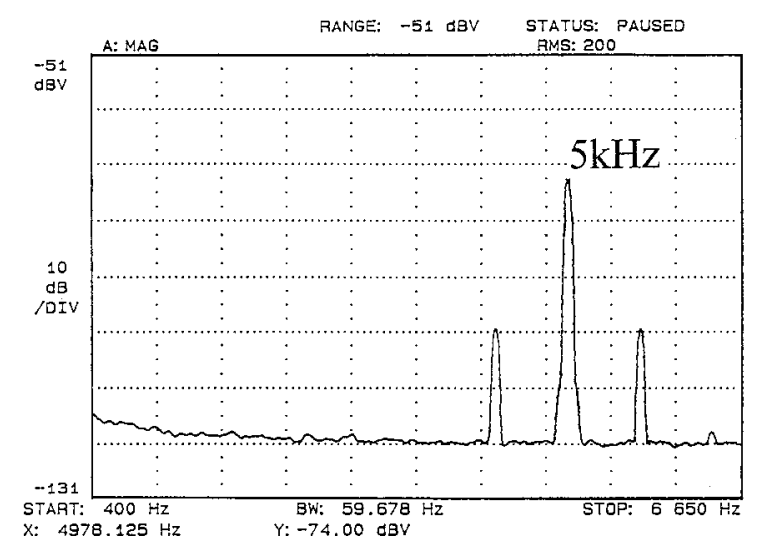

Fig. 3. Spectrum analyzer trace recorded with incident sound waves at $700 \mathrm{~Hz}$.

that the sideband frequency components are symmetric about the $(5-\mathrm{kHz})$ carrier signal: this frequency spectrum is an expected system response. Similar results were also obtained by use of sound waves of frequencies as high as a few kilohertz and PZTinduced strains of frequencies between 10 and 20 $\mathrm{kHz}$. We also modulated the grating in water by using an unfocused $76-\mathrm{kHz}$ transducer and again observed the same expected system response. We conclude therefore that for this range of modulation frequencies the optical system is functioning as expected.

Now consider Fig. 4, which shows a typical spectrum analyzer trace recorded with the focused 1.91$\mathrm{MHz}$ transducer. Two striking anomalies in the response of the system can be seen: first, note that the upper and lower sideband frequency components are no longer symmetric; and second, note the existence of a large homodyne signal at $1.91 \mathrm{MHz}$, which was as much as several decibels greater than the sideband magnitudes. We also found that the sidebands did not fall to zero as the OPD between the two arms of the MZI approached zero, but remained finite and were of equal magnitude. ${ }^{21}$

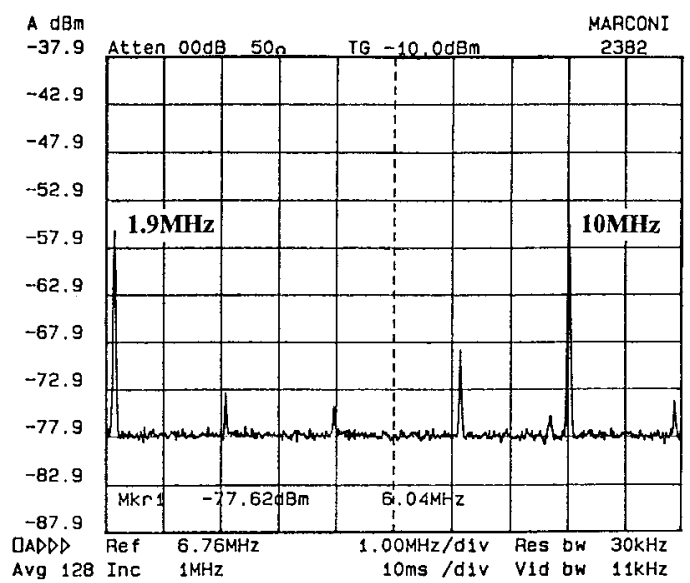

Fig. 4. Spectrum analyzer trace recorded with focused ultrasound at $1.91 \mathrm{MHz}$.

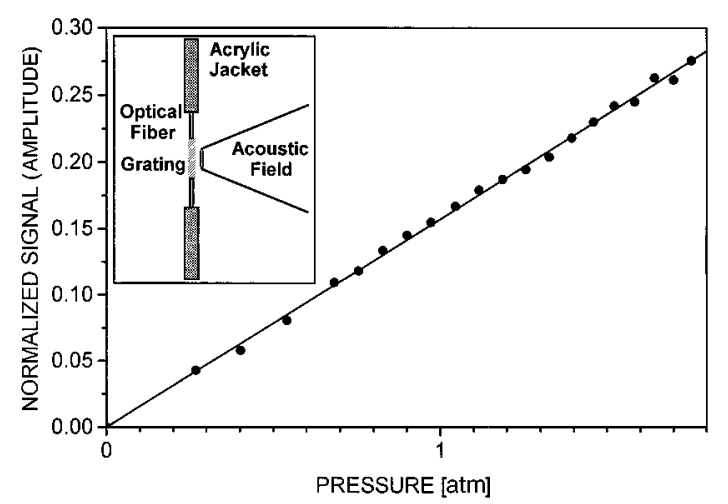

Fig. 5. Sideband magnitude (normalized to carrier magnitude) as a function of incident acoustic pressure.

Despite these anomalies, we recorded the results in Fig. 5, which shows the detected magnitude of one of the sidebands normalized by its corresponding carrier signal (to compensate for any intensity fluctuations of light levels in the system) as a function of acoustical pressure incident on the IFBG. As can be seen, a linear response is demonstrated. We also recorded the response of the homodyne signal to the acoustical pressure. This too gave a linear response and was particularly encouraging because the homodyne signal exhibits a greater magnitude than the sideband signal (for the same acoustical pressure) and so potentially could offer an improved noiselimited pressure resolution.

Based on these preliminary data, it would seem that the IFBG could be successfully used as an ultrasonic probe. However, consider now Fig. 6, in which we scanned the focal spot of the transducer along the fiber-grating and recorded the dependence of one of the sideband powers (normalized by its corresponding carrier power) on displacement. Note the multiple peaks and troughs in the system response, which are observed over a distance that is much greater than the grating length. In addition, we also monitored the homodyne signal during the scan and (as will be shown shortly) observed similar profiles with focal-spot displacement. Again, these are unexpected results because the radius of the focal spot is

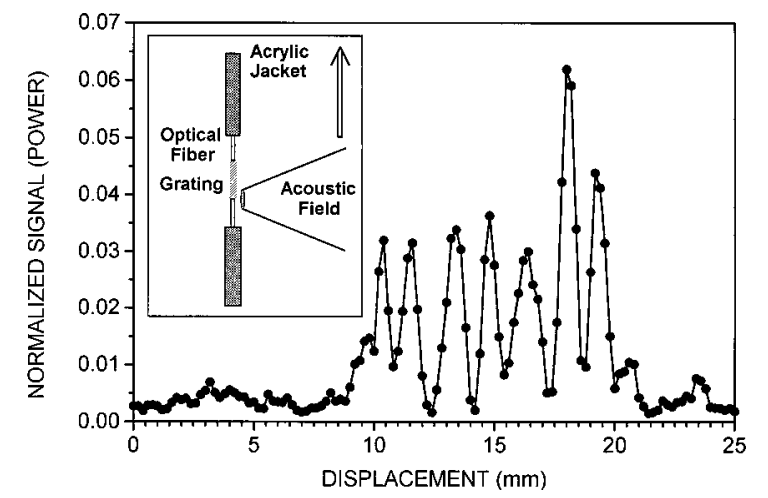

Fig. 6. Sideband power (normalized to carrier power) as a function of longitudinal IFBG position. 
only approximately $1 \mathrm{~mm}$. Finally, we replaced the IFBG with an ordinary fiber and placed that at the focal region of the transducer. As expected, we observed no frequency spectra, thus confirming that any signals we do observe stem from the grating alone. It is evident therefore from these data that the IFBG studied exhibits insufficient longitudinal resolution and that the sensitivity of the system is periodic and highly dependent on the position of the focal spot on the fiber. These factors thus preclude this IFBG (on its own) from being used as an ultrasonic probe.

\section{Standing Waves and Nonuniform Strain}

Consider Fig. 6 and the average distance between the peak responses, which was measured to be $1.48 \mathrm{~mm}$. If we hypothesize that the acoustic coupling from the ultrasonic field to the optical fiber leads to the formation of standing waves in the fiber, then this value leads to an experimental acoustic wavelength of 2.96 $\mathrm{mm}$, which is close to the predicted value of $3.09 \mathrm{~mm}$ for compressional waves at $1.91 \mathrm{MHz}$ in fused quartz. We repeated these experiments, this time driving the transducer at $1.6 \mathrm{MHz}$, and found an experimental value of $3.76 \mathrm{~mm}$, which is again close to the predicted value of $3.68 \mathrm{~mm}$ for compressional waves.

We thus propose the following explanation: compressional standing waves, which can extend many centimeters, are set up by the ultrasound in the fiber. These give rise to periodic maximum and minimum longitudinal displacements in the region of the IFBG as the focal spot is moved along the fiber, which we observed as the peaks and troughs in the system response. As to the origin of these waves, we further postulate that, because the fiber is fixed to the frame (see Fig. 2) many centimeters away from the IFBG, and also because (as we shortly show) the acrylic jackets on either side of the grating tend to attenuate these acoustic modes, it is likely that the standing waves stem from partial backreflections at the bare optical fiber-acrylic jacket boundary.

Based on this premise of standing waves, and in particular standing waves that here are shorter than the grating length, an important consequence would be that the IFBG is subject to a nonuniform strain; sections of the grating are unmodulated, and other modulated sections are out of phase with each other. The grating now behaves in a manner similar to that of smaller separate gratings that are adjacent to each other, each of which backreflects light of slightly different wavelengths. The result is that various regions of the IFBG act as spectral filters for the backreflected light from other regions of the IFBG and so give rise to amplitude modulations. (This is analogous to sensing schemes in which a reading grating is used to convert a modulated wavelength shift from a sensing grating into an amplitude modulation. ${ }^{17}$ To validate this hypothesis we shone light directly onto the grating (through a coupler) without the interferometer present and observed a signal at $1.91 \mathrm{MHz}$ (plus additional smaller harmonics). Hence the homodyne signal observed by use of the interferometric scheme stems from an amplitude variation with, in this case, a measured modulation depth of a few percent.

To model what effect an amplitude modulation would have on the interferometric signals, we can now replace the constant $A$ in Eq. (1) (in the simplest case) with

$$
A[(1-b)+b \cos (\omega t+\varphi)]
$$

where $b$ is a modulation index and $\varphi$ is a phase term. As we showed in Ref. 21, solving for the frequency spectra for this modified variant of Eq. (1) leads to finite symmetric sidebands at zero OPD and asymmetric sidebands for nonzero OPD's, as observed in experiment. In addition, it is straightforward to show that, for a small $b$ with a linear dependence on acoustic pressure (as was found), we would expect the sideband magnitudes to exhibit also a linear dependence with acoustic pressure (as we again found).

In summary, the proposed standing waves that are set up in the fiber lead to a nonuniform strain in the grating, which in turn gives rise to an amplitude modulation. The amplitude modulation that we observe as the homodyne signal modifies the interferometric signals, resulting in the asymmetric sideband magnitudes.

We finally note that the wavelength of the lowerfrequency sound waves (ranging from meters to centimeters) is in all cases much greater than the grating length. Hence in these cases the grating is subject to a more uniform strain, and so none of the anomalies in the system response were observed.

\section{Desensitization of Fiber}

The system discussed in Subsections 3.A.1 and 3.A.2 exhibits a linear response with acoustic pressure. However, as we mentioned earlier, it is clear from Fig. 6 that the IFBG on its own cannot be used as a probe because it exhibits insufficient longitudinal resolution.

Consider now Figs. 7(a) and 7(b), in which we performed longitudinal scans on the same grating but with different interjacket separations, and recorded the homodyne response with focal-spot displacement. (Similar profiles were also observed for the sideband response.) In Fig. 7(a) the interjacket separation was $\approx 1 \mathrm{~cm}$, and in Fig. 7(b) it was $\approx 6 \mathrm{~cm}$. Note that in each case the peaks and troughs in the system response extend over approximately the same distances as the respective interjacket separation, thus indicating that the acrylic jackets, which are on either side of the IFBG, can significantly attenuate the acoustic waves in the fiber. Based on this observation, it is apparent that the longitudinal resolution may be improved by an appropriate desensitization of the optical fiber to the acoustical field.

To achieve this desensitization, we initially coated the fiber with acrylic compounds (of diameters $<1$ $\mathrm{mm}$ ) and various resins (including resin mixed with tungsten powder, which is known to attenuate acoustic fields ${ }^{22}$ ), but leaving a 1-mm gap in the viscinity of 

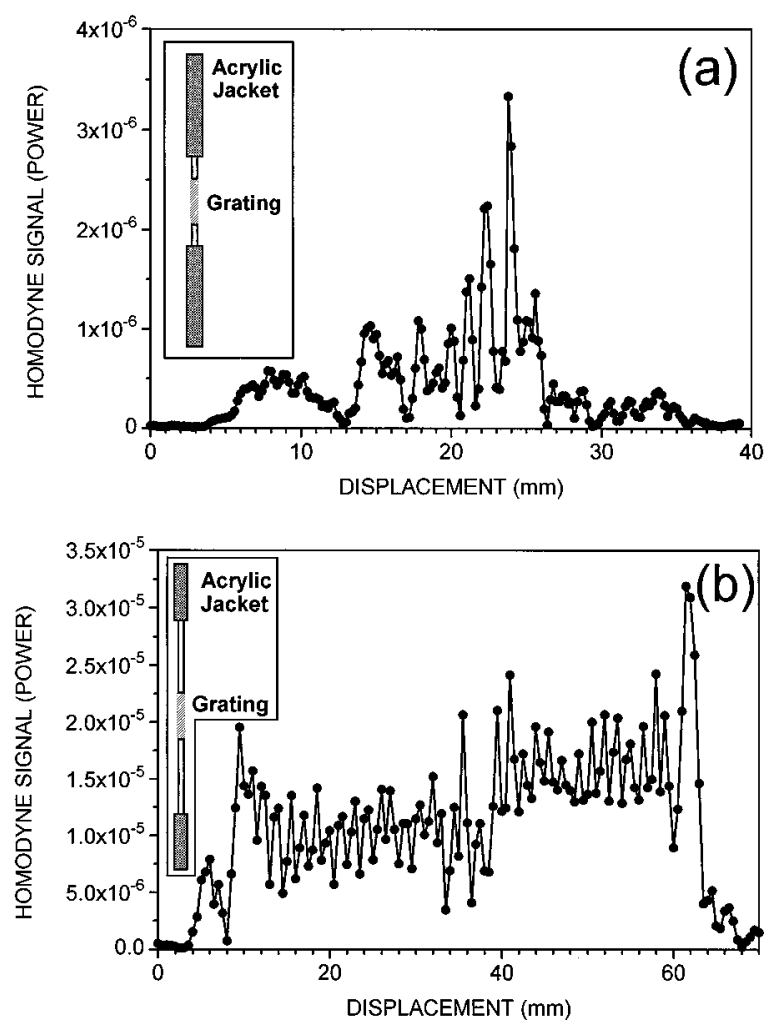

Fig. 7. Sideband power (normalized to carrier power) as a function of longitudinal IFBG position for an interjacket separation of (a) $\approx 1 \mathrm{~cm}$ and $(\mathrm{b}) \approx 6 \mathrm{~cm}$.

the IFBG. A typical longitudinal scan of one of these arrangements is shown in Fig. 8. As can be seen, although the resolution has improved, this method was not entirely successful: first, it is evident that, although attenuated, the ultrasound can still significantly penetrate the coating; and second, extended periods of time under the acoustic pressures used here (which are in fact relatively small compared with some medical applications of ultrasound) resulted in the coatings being cleaned off.

We found that a more successful approach to desensitization was to jacket the fiber with polyvinyl chlo-

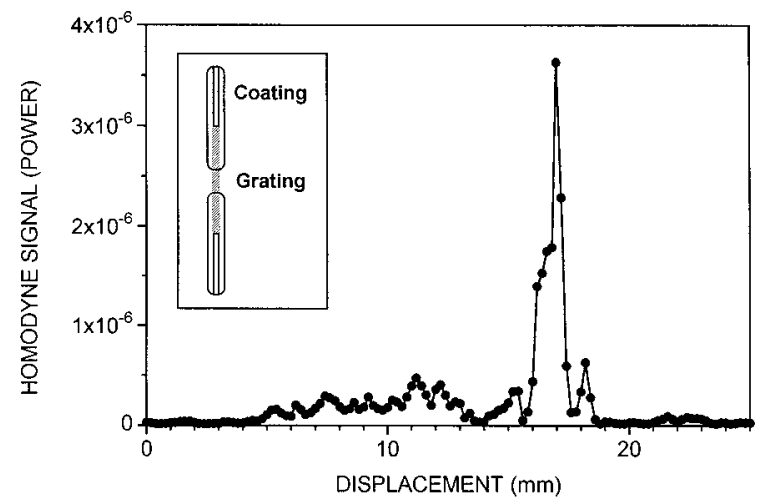

Fig. 8. Sideband power (normalized to carrier power) as a function of longitudinal IFBG position for fiber coated with resin mixed with tungsten powder.
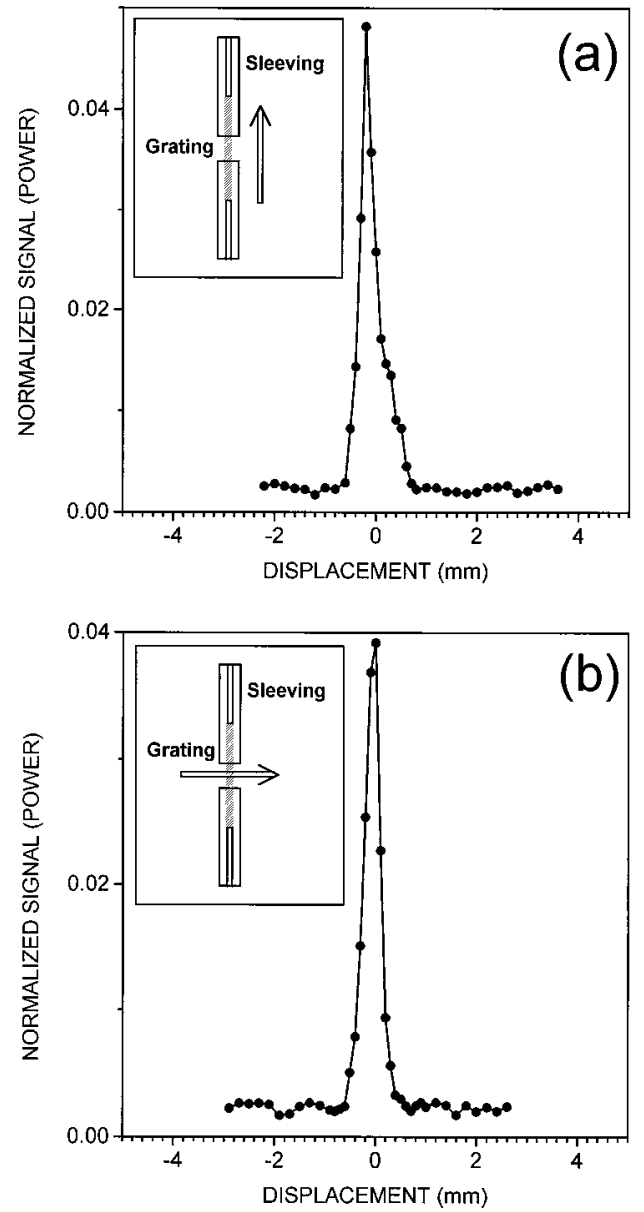

Fig. 9. Sideband power (normalized to carrier power) for the sleeved IFBG as a function of (a) longitudinal and (b) lateral IFBG position.

ride (PVC) sleeving. Here we used sleeving of 0.88-mm outer diameter in which the internal spacing between the bare optical fiber and the sleeving was $\approx 50 \mu \mathrm{m}$. A gap in the PVC allowed $\approx 1 \mathrm{~mm}$ of IFBG to be exposed to the acoustic field. The results of a longitudinal scan and a lateral scan of the acoustical focal spot are shown in Figs. 9(a) and 9(b), respectively. As can be seen, the improvements are significant although the profiles are a little narrower than would be expected. This we comment on shortly. In addition, when the 1-mm gap in the PVC was moved a few millimeters to one side of the IFBG, the system response fell to near noise level, indicating that the acoustic modes in the fiber were significantly attenuated by the jacket. This result is encouraging if a probe containing more than one IFBG multiplexed onto the same fiber is to be designed.

However, although the resolution of the IFBG is improved, consider now Fig. 10, which shows the sideband dependence with the incident acoustic pressure using this shielded arrangement. Note that the response is nonlinear and that this nonlinearity is likely to have narrowed the profiles shown in Figs. 9(a) and 9(b). In addition, we also recorded the homodyne dependence with acoustic pressure and 


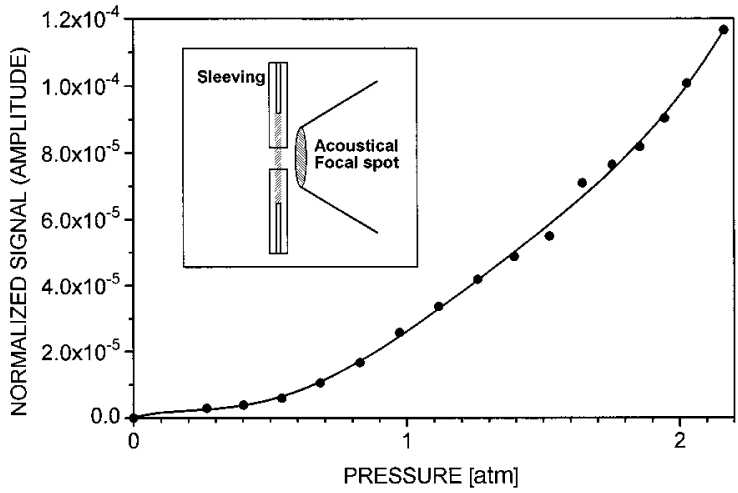

Fig. 10. Sideband magnitude (normalized to carrier magnitude) as a function of incident acoustic pressure for the sleeved IFBG.

again found a nonlinear response that, in this case, did not increase monotonically with pressure.

It is evident therefore that the sleeving has a detrimental effect on the response of the grating. Figure 11 shows why: the spectra of the backreflected light from the grating (recorded with an optical spectrum analyzer that averages the spectra over a time interval of approximately $1 \mathrm{~s}$ ) is asymmetric in response to the acoustic field and indicative of light from various regions of the grating having undergone significant static wavelength shifts. This implies that, as discussed earlier, regions of the grating act as spectral filters and so again give rise to amplitude modulations that modify the interferometric signals (although here they are not linear with acoustic pressure, as evidenced by the homodyne response).

We found that these asymmetric profiles were due to a localized heating of the PVC by the incident acoustic energy. We confirmed this by placing a sample of PVC sleeving at the acoustical focal spot and measuring the temperature in its viscinity using a small thermocouple. Temperature rises of approx-

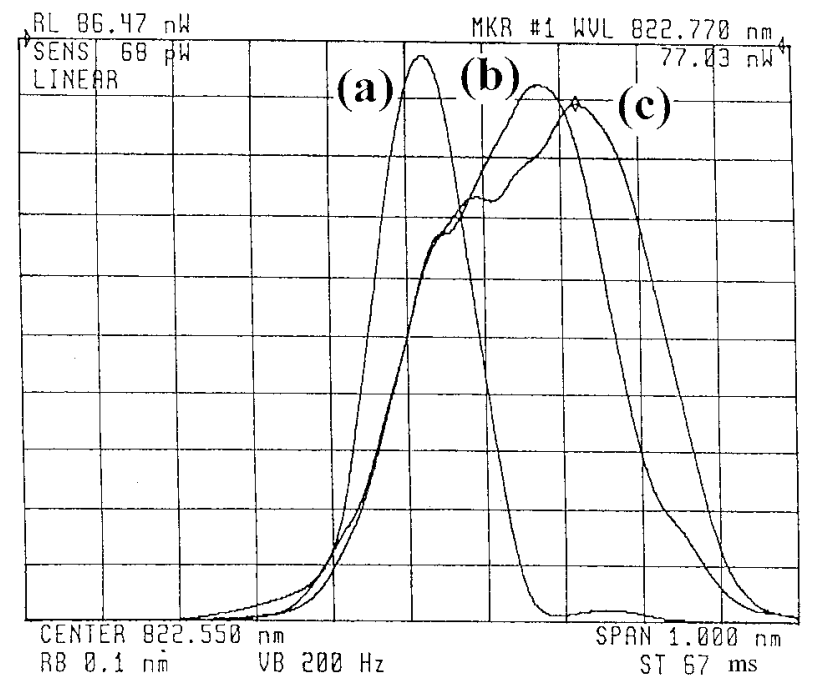

Fig. 11. Wavelength spectra of backreflected light from the sleeved IFBG for incident acoustic pressures of (a) $0 \mathrm{~atm}$, (b) 1.4 atm, (c) $2.2 \mathrm{~atm}$. imately $10{ }^{\circ} \mathrm{C}$ were recorded. Hence, because the grating is 5-mm long with only $1 \mathrm{~mm}$ exposed to the acoustical field at the ambient water temperature, it is evident this heating leads to a nonuniform temperature gradient across the IFBG.

We thus conclude the following: we successfully localized the longitudinal response of the IFBG to the acoustical field, but in doing so we generated a new problem, namely, a nonuniformity in temperature across the grating. For the grating to function correctly (in terms of applying both a uniform strain and a uniform temperature) we must now consider shorter gratings.

\section{B. 1-mm Grating}

Based on our hypothesis, it is apparent that the grating length should be made smaller than half the acoustic wavelength in fused quartz for the grating to be subject to an approximate uniform strain. To demonstrate this we took a standard 5-mm-long grating and gradually removed small pieces of it from one end until approximately only $1 \mathrm{~mm}$ of grating remained. As each piece was removed we recorded the system response to the ultrasonic field using the shortened grating. The results are shown in Fig. 12. Note the dramatic decrease in the homodyne signal (which falls to rf interference levels), along with the more symmetric sideband magnitudes. In addition, despite the expected decrease in the backreflected light intensity as the grating is shortened, it is interesting to note that there is an initial significant increase in the sideband magnitudes. Based on the stationary wave model, it seems likely that, with the use of long gratings, sections of the grating may well have been modulated in antiphase with each other. The interference signals originating from these sections would also have been in antiphase, and so a canceling-out effect occurs. As the grating is diminished this effect lessens, resulting in the increased sideband magnitude that we observed.

Again, because of the results of Fig. 6, it is apparent that this shortened grating on its own cannot be used as a high-frequency probe since it exhibits insufficient longitudinal resolution. Hence we once more jacketed the fiber with PVC sleeving such that only the 1-mm grating at the end of the fiber was exposed to the acoustical field. The results of a longitudinal scan and a lateral scan of the focal spot are shown in Figs. 13(a) and 13(b), respectively. These data compare favorably with the diameter of the main diffraction maximum $(2.3 \mathrm{~mm})$ of the transducer, although there is evidence of small scattering effects near the grating-sleeving boundary.

Figure 14 shows the detected magnitude of one of the sidebands (normalized by its corresponding carrier signal) as a function of the acoustical pressure incident on the grating. It is clear that the system response is now linear, and (for this probe) we determined a noise-limited pressure resolution of $\approx 4.5 \times$ $10^{-3} \mathrm{~atm} / \mathrm{V} \mathrm{Hz}$, which corresponds to a noise-limited intensity resolution of approximately $1 \times 10^{-3}$ $\mathrm{Wcm}^{-2} \mathrm{~Hz}^{-1 / 2}$.

We finally show in Fig. 15 the spectra of the back- 


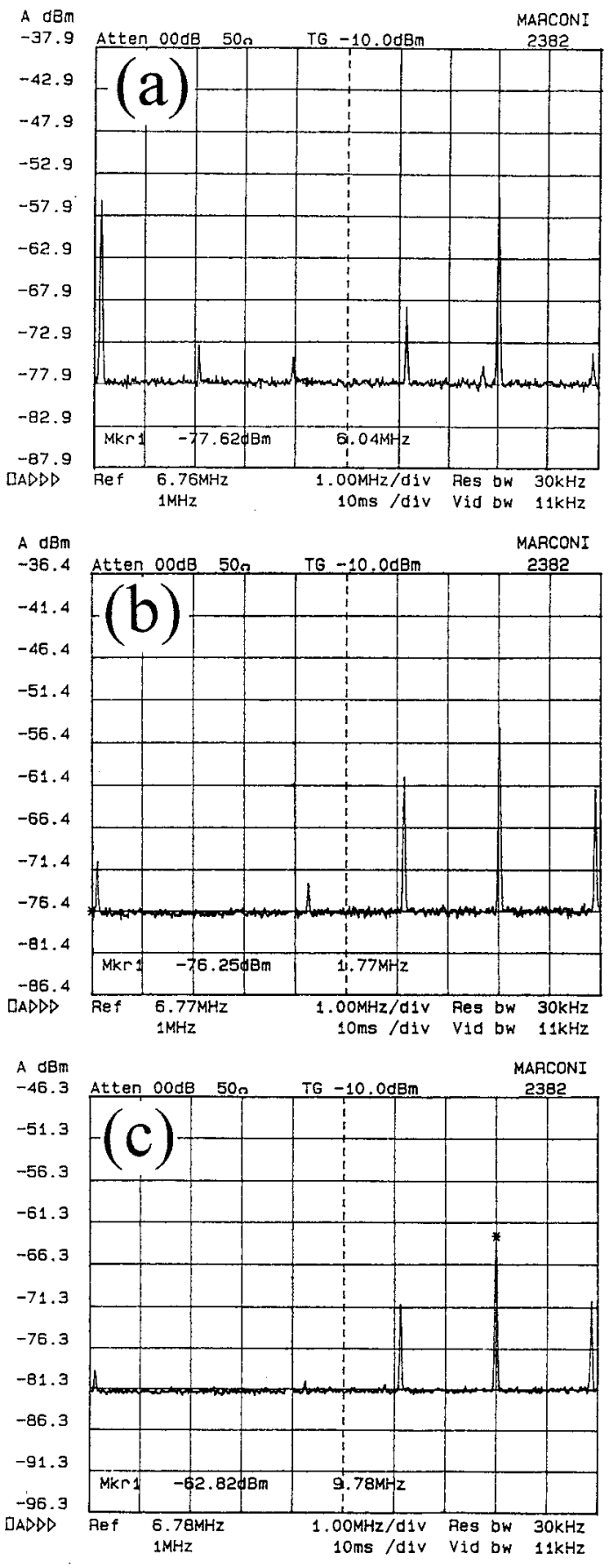

Fig. 12. Spectrum analyzer trace recorded with focused ultrasound at acoustic pressure of $\approx 2$ atm for the (a) $\approx 5$-mm grating, (b) $\approx 3$-mm grating, $(\mathrm{c}) \approx 1$-mm grating.

reflected light from the shielded 1-mm grating in response to acoustic pressure. The profiles are symmetric and so indicate that the grating is now subject to a uniform temperature gradient across its length and hence is behaving as expected.

\section{General Discussion}

The improvement in the system response with the use of a short grating is significant. However, several points should be noted.
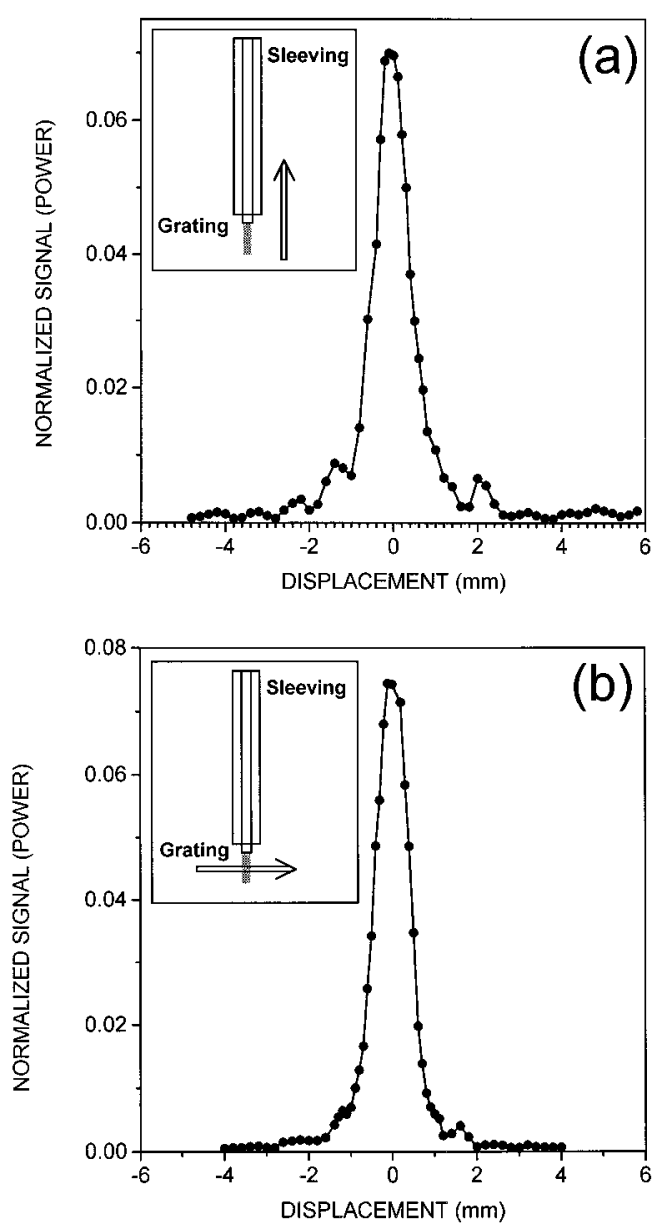

Fig. 13. Sideband power (normalized to carrier power) for the sleeved 1-mm IFBG as a function of (a) longitudinal and (b) lateral IFBG position.

First, in our experiment by shortening the grating we dramatically reduced its reflectivity (in this case by a factor of well over 100) as well as increased its bandwidth, and so we limited its noise-limited resolution. For applications involving high-power ablation in which pulsed ultrasound intensities can be several or even thousands of watts per centimeter,

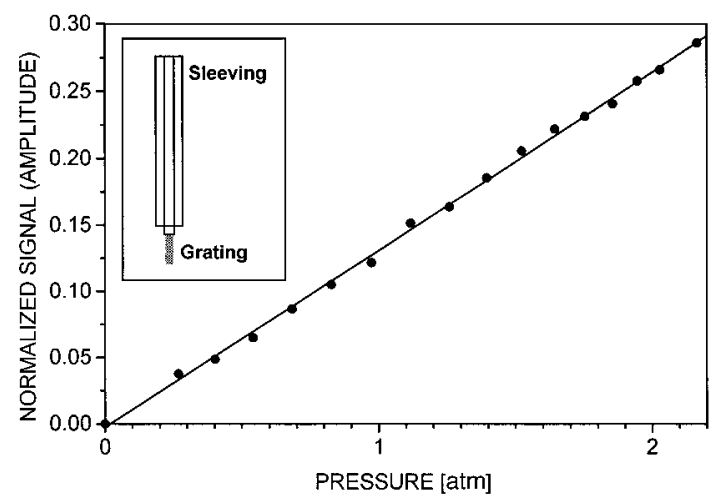

Fig. 14. Sideband magnitude (normalized to carrier magnitude) as a function of incident acoustic pressure for the sleeved 1-mm IFBG. 


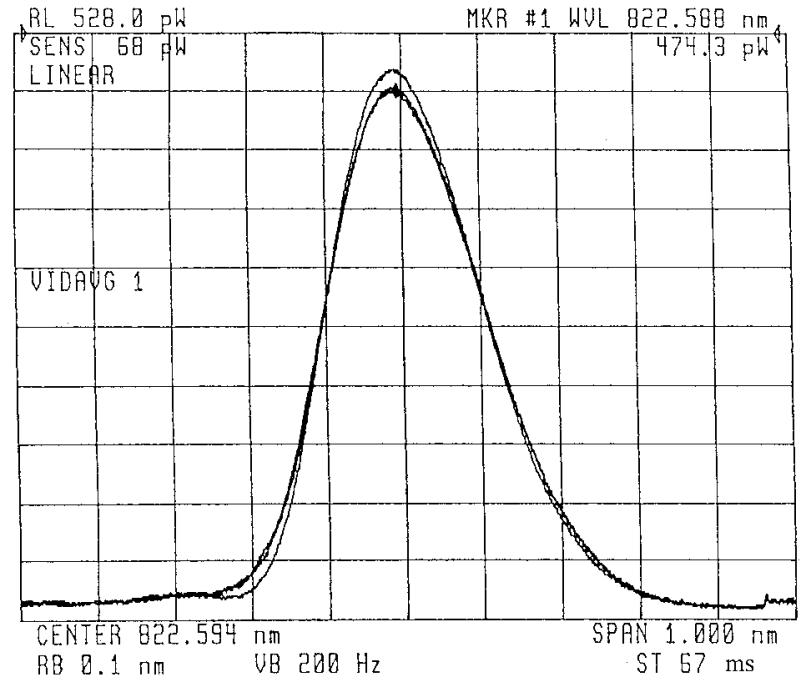

Fig. 15. Wavelength spectra of backreflected light from the sleeved 1-mm IFBG for incident acoustic pressures of 0, 1.4, and $2.2 \mathrm{~atm}$. Note that the profiles are now almost identical, which was not the case in Fig. 11.

the resolution we obtained is sufficient. For physiotherapy treatments in which the acoustic intensities can vary from approximately 0.2 to $2 \mathrm{Wcm}^{-2}$, an improvement may well be necessary. Gratings, however, of lengths less than $1 \mathrm{~mm}$ but with $90 \%$ reflectivity have been manufactured, and so we anticipate a greatly improved pressure resolution with the use of such gratings.

Second, based on the stationary wave model, a limiting factor in the successful performance of the grating is that its length should be less than half the acoustic wavelength in fused quartz. This sets an upper limit on the incident acoustic frequency that can be measured. Fortunately, frequencies of between $500 \mathrm{kHz}$ and $4 \mathrm{MHz}$ are generally used in most medical applications of ultrasound, which implies that the grating lengths should be no greater than approximately $0.5 \mathrm{~mm}$ for the highest frequency. Again, such lengths are available. In the case of lithotripsy, multiple-frequency components may be generated. However, as the acoustic field traverses several centimeters of tissue, the higher-order modes are in general significantly attenuated. Hence in certain cases acoustic fields of only a few megahertz may be of importance. A final though unrelated point entails the grating acting as an acoustic probe in smart-structure design. Here acoustic emission is monitored and gives information about twinning, dislocation motion, cracking, etc., in the material. ${ }^{23}$ Of course, the response of the grating to multifrequency bursts needs to be investigated. However, typical frequency spectra range from approximately 0.1 to $1.0 \mathrm{MHz}$, and so readily available gratings of lengths of 1-2 mm can (in principle) be used.

Third, more sophisticated techniques (entailing multiple coatings) for desensitizing optical fibers have been reported ${ }^{24}$ : In our experiments we observed fluctuations in the rf power (to the transducer) as the IFBG sensor was moved around in the vicinity of the focal spot. This was especially evident when the focal spot was incident on the PVC sleeving and is evidence for backreflections and scattering of the acoustical field by the sensor. Obviously, reducing the dimensions of the shielding by using these multiple coatings greatly minimizes this scattering. In addition, it is worth mentioning again that, if the acoustic modes in the fiber can be significantly attenuated using these coatings (as we found using the sleeving), then in principle it should be possible to multiplex IFBG's onto the same fiber with little or no acoustic cross talk between them.

Finally, as far as we are aware, this is the first time that data indicating the presence of acoustic stationary waves in the fiber have been reported. Because the IFBG sensor is localized in the fiber, we can see evidence for this in our longitudinal scans. In some other interferometric and polarimetric schemes the sensing element is the entire fiber length. Sensing is therefore not localized, and so in performing longitudinal scans evidence for the stationary waves may not be readily observable.

\section{Conclusions}

We have demonstrated that a Bragg grating can function effectively as a point ultrasonic probe with millimeter resolution if (a) the grating length is small (less than half the acoustic wavelength in fused quartz) and (b) the fiber is appropriately desensitized. A distinct advantage this probe has over some other optical sensors is also its potential to measure temperature simultaneously. This is a critical factor in hyperthermia treatments, in which the temperature distribution in tumors and cancers needs to be monitored. Schemes using IFBG's for temperature measurements only in such applications have been well demonstrated, ${ }^{25}$ and we have recently carried out preliminary research to investigate the simultaneous recovery of temperature and ultrasonic field. ${ }^{26}$

\section{References}

1. W. L. Nyborg, "Optimization of exposure conditions for medical ultrasound," Ultrasound Med. Biol. 11, 245-260 (1985).

2. National Council on Radiation Protection and Measurements, "Biological effects of ultrasound: mechanisms and clinical implications," NCRP Rep. No. 74 (National Council on Radiation Protection and Measurements, Bethesda, Md., 1983).

3. B. B. Barnett, G. R. ter Haar, M. C. Ziskin, W. L. Nyborg, K. Maeda, and J. Bang, "Current status of research on biophysical effects of ultrasound," Ultrasound Med. Biol. 20, 205-218 (1994).

4. N. T. Sanghvi, F. J. Fry, R. Bihrle, R. S. Foster, M. H. Phillips, J. Syrus, A. V. Zaitsev, and C. W. Hennige, "Noninvasive surgery of prostate tissue by high-intensity focused ultrasound," IEEE Trans. Ultrason. Ferroelectr. Freq. Control 43, 10991110 (1996)

5. C. R. Hill, "Optimum acoustic frequency for focused ultrasound surgery," Ultrasound Med. Biol. 20, 271-277 (1994).

6. S. B. Field and J. W. Hand, eds., An Introduction to the Practical Aspects of Clinical Hyperthermia (Taylor and Francis, London, 1990).

7. "Guidelines for the safe use of extra corporeal shock-wave lithotripsy (ESWL) devices," in Proceedings of the Radiation 
Safety Committee of the European Federation of Societies for Ultrasound in Medicine and Biology, Ultrasound Med. Biol. 20, 315-316 (1994).

8. R. P. De Paula, L. Flax, J. H. Cole, and J. A. Bucaro, "Single mode fiber ultrasonic sensor," IEEE J. Quantum Electron. 18, 680-693 (1982).

9. S. Knudsen and K. Blotekjaer, "An ultrasonic fiber-optic hydrophone incorporating a push-pull Sagnac interferometer," J. Lightwave Technol. 12, 1696-1700 (1994).

10. H. L. W. Chan, K. S. Chiang, D. C. Price, and J. L. Gardener, "The characterisation of high frequency ultrasonic fields using polarimetric optical fiber sensor," J. Appl. Phys. 66, 1565-1570 (1989).

11. H. L. W. Chan, K. S. Chiang, D. C. Price, J. L. Gardener, and J. Brinch, "Use of a fiber-optic hydrophone in measuring acoustic parameters of high power hyperthermia transducers," Phys. Med. Biol. 34, 1609-1622 (1989).

12. K. S. Chiang, H. L. W. Chan, and J. L. Gardener, "Detection of high frequency ultrasound with polarisation maintaining fiber,” J. Lightwave Technol. 8, 1221-1227 (1990).

13. P. C. Beard and T. N. Mills, "Optical fiber sensor for the detection of laser-generated ultrasound in arterial tissues," in Medical Sensors II and Fiber Optic Sensors, A. M. Verga Scheggi, F. Baldini, P. R. Coulet, and O. S. Wolfbeis, eds. Proc. SPIE 2331, 112-122 (1994).

14. J. J. Alcoz, C. E. Lee, and H. F. Taylor, "Embedded fibre-optic Fabry-Perot ultrasound sensor," IEEE Trans. Ultrason. Ferroelectr. Freq. Control 37, 302-305 (1990).

15. P. C. Beard and T. N. Mills, "Miniature optical fibre ultrasonic hydrophone using a Fabry-Perot polymer film interferometer," Electron. Lett. 33, 801-803 (1997).

16. P. St. J. Russell, J.-L. Archambault, and L. Reekie, "Fibre gratings," Phys. World 6, 41-46 (1993).
17. Y.-J. Rao, "In-fibre Bragg grating sensors," Meas. Sci. Technol. 8, 355-375 (1997).

18. D. A. Jackson, A. D. Kersey, M. Corke, and J. D. C. Jones, "Pseudo-heterodyne detection scheme for optical interferometer," Electron. Lett. 18, 1081-1083 (1982).

19. A. D. Kersey, T. A. Berkoff, and W. W. Morey, "Fiber-optic grating strain sensor with drift-compensated high-resolution interferometric wavelength-shift detection," Opt. Lett. 18, 72-74 (1993).

20. G. Kossoff, "Analysis of focusing action of spherically curved transducers," Ultrasound Med. Biol. 5, 359-365 (1979).

21. N. E. Fisher, S. F. O’Neill, D. J. Webb, C. N. Pannell, D. A. Jackson, L. R. Gavrilov, J. W. Hand, L. Zhang, and I. Bennion, "Response of in-fibre Bragg gratings to focused ultrasonic fields," in Proceedings of the 12th International Conference on Optical Fiber Sensors, G. W. Day and A. D. Kersey, eds. (Optical Society of America, Washington, D.C., 1997), pp. 190193.

22. C. M. Sayers and C. E. Tait, Ultrasonic Properties of Transducer Backings (Butterworth, Oxford, UK, 1984), pp. 57-60.

23. J. Kaiser, "Investigation of acoustic emission in tensile testing," Ph.D dissertation (Technische Hochscule, Munich, Germany, 1950).

24. N. Lagakos, G. Ku, J. Jarzynski, J. H. Cole, and J. A. Bucaro, "Desensitization of the ultrasonic response of single-mode fibers," J. Lightwave Technol. 5, 1036-1039 (1985).

25. Y. Rao, D. J. Webb, D. A. Jackson, L. Zhang, and I. Bennion, "In-fibre Bragg-grating temperature sensor system for medical applications," J. Lightwave Technol. 15, 779-785 (1997).

26. N. E. Fisher, D. J. Webb, C. N. Pannell, D. A. Jackson, L. R. Gavrilov, J. W. Hand, L. Zhang, and I. Bennion, "Ultrasonic field and temperature sensor based on short in-fibre Bragg gratings," Electron. Lett. 34, 1139-1140 (1998). 